\title{
The Dusty, Solar Type Spectroscopic Binary BD +20 307
}

\author{
Francis C. Fekel ${ }^{*}$, B. Zuckerman ${ }^{\dagger}$, Michael H. Williamson*, \\ Gregory W. Henry* and M. P. Muno** \\ ${ }^{*}$ Center of Excellence in Information Systems, Tennessee State University, Nashville, TN \\ ${ }^{\dagger}$ Department of Physics and Astronomy, University of California, Los Angeles, CA \\ ${ }^{*}$ Space Radiation Laboratory, California Institute of Technology, Pasadena, CA
}

\begin{abstract}
The dustiest known main-sequence star, BD +20307 , is actually a double-lined binary with a period of 3.4202 days and a circular orbit. The system is also metal poor with $[\mathrm{Fe} / \mathrm{H}]=-0.4$. The components are late-F and early-G dwarfs and have a mass ratio of 1.07. The photometric period of about 3.5 days indicates that the components are synchronously rotating. The metal poor, binary nature invalidates the idea that the object is a very young single star with a warm planetmaking dust disk. Instead, the metal poor nature of the system and the lithium abundances of the components argue that the system is likely several billion years old, and so the dust disk results from the recent collision of two planetary mass rocky objects. Thus, BD +20 307 may well be the first known system with planets orbiting a close binary star.
\end{abstract}

Keywords: planetary systems: formation — stars: individual(BD +20 307) — binaries: spectroscopic - stars: rotation

PACS: $97.80 . \mathrm{Fk}, 97.82 . \mathrm{Jw}, 97.10 . \mathrm{Qh}$

\section{INTRODUCTION}

As indicated by infrared observations, the debris disk of the field star BD +20 307 contains warm dust analogous to that in the Sun's asteroid belt. This suggests that the system had a recent collision of two planetary embryos or planets in the terrestial planet zone (Rhee et al. 2008). Presumed to be a single star, Song et al. (2005) estimated an age of $300 \mathrm{Myr}$ from several age indicators. To refine their estimate we obtained Xray measurements with the Chandra X-ray Observatory and photometric observations. However, Weinberger (2008) recently showed that BD +20 307 is a 3.4 day binary, consisting of two similar stars. Thus, we have also obtained radial velocity observations to refine the properties of the stars. With the discovery that the star is a short-period binary, the X-ray measurements and the photometric observations no longer provide significant constraints on the system's age. Here, we primarily discuss the spectroscopic and photometric observations and what they tell us about this binary system.

\section{SPECTROSCOPIC RESULTS}

The spectroscopic observations were obtained at Fairborn Observatory near Washington Camp in southeast Arizona. In 2008 we obtained 28 echelle spectrograms (wavelength range 4920-7100 $\AA$, resolution of $0.17 \AA$, $\mathrm{S} / \mathrm{N} \sim 10$ ) with the $2 \mathrm{~m}$ automatic telescope 


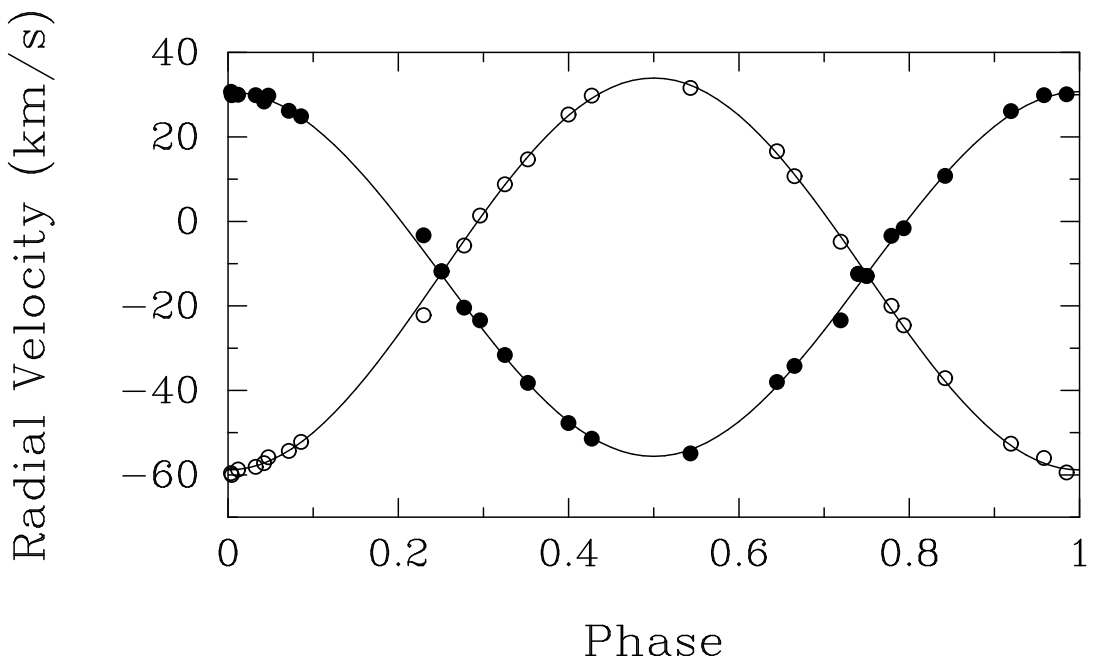

FIGURE 1. Radial velocities (filled circles $=$ component $\mathrm{A}$, open circles $=$ component $\mathrm{B}$ ) of $\mathrm{BD}+20$ 307 compared with the computed radial velocity curves. Zero phase is a time of maximum velocity of the primary, component A.

and measured the radial velocities of the components. Figure 1 shows the resulting circular orbit that has a period of $3.4202 \pm 0.0007$ days. The mass ratio is 1.07 , and the $V$ magnitude difference, determined from the difference in line strengths of the two components, is $0.27 \pm 0.1$. Comparing stars with known abundances to the $\mathrm{BD}+20307$ system, we estimate $[\mathrm{Fe} / \mathrm{H}]=-0.4$, making the components metal poor relative to the Sun. The combined color of the stars, the magnitude difference, and the parallax indicate that the components of BD +20 307 are a late-F and an early-G dwarf. Masses for such stars compared with the minimum masses suggest an orbital inclination of $\sim 30 \mathrm{deg}$.

\section{PHOTOMETRIC RESULTS}

Differential photometric Strömgren $b$ and $y$ observations were obtained at Fairborn Observatory with a $0.8 \mathrm{~m}$ automatic telescope over four seasons. When analyzed season by season or as a whole, the data showed no periodicity. However, visual inspection of the light curves revealed that the system does sometimes show low-amplitude, coherent brightness variability. Analysis of the most coherent section of the data produced a period of $3.530 \pm 0.025$ days (Figure 2). Other portions of the data yielded less precise period values of 3.28 and 3.44 days. These light variations result from a non-uniform distribution of spots that rotate in and out of view, providing a determination of the rotation period. A short-period solar-type binary is expected to have synchronous rotation, and the photometric periods are indeed in good agreement with the spectroscopic period. 

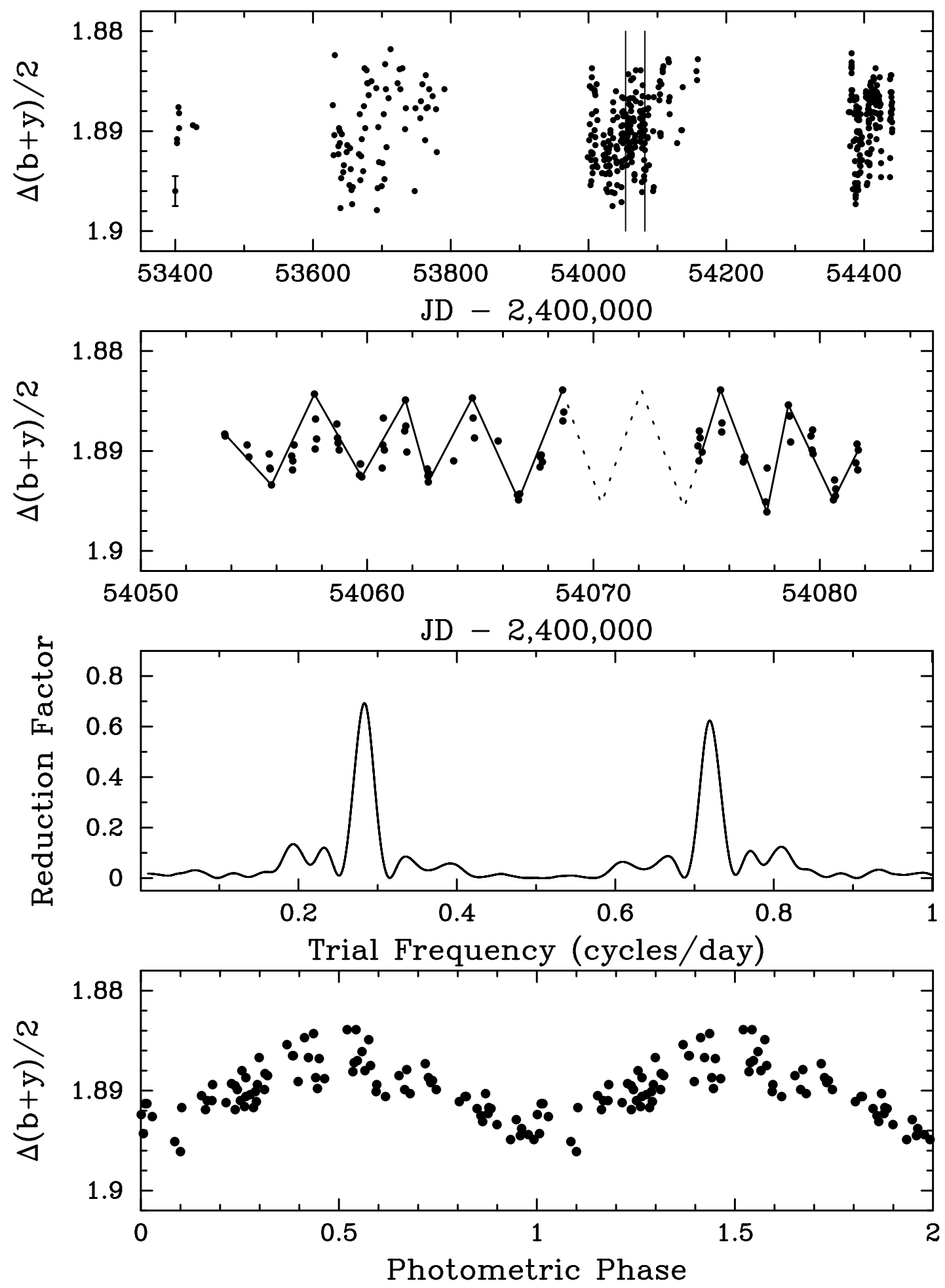

FIGURE 2. Top panel: Photometric observations taken in the Strömgren $(b+y) / 2$ passband over four observing seasons. Second panel: Part of the third observing season that shows low-amplitude coherent variability. Third panel: Power spectrum of the second panel data. Best period is 3.530 days. Bottom panel: Coherent data phased with the 3.530 day period. 


\section{DISCUSSION}

Although the solar-type components have similar properties, differing by only $7 \%$ in mass and having a $V$ mag difference of just 0.3 , Weinberger (2008) found that the lithium equivalent width of the secondary was not measureable, and thus, at least 4 times less than that of the primary. Lithium comparisons with late-F and early-G stars in various clusters indicate that the system's age is at least $1-2$ Gyr. The low metallicity of $[\mathrm{Fe} / \mathrm{H}]$ $=-0.4$ also suggests an age of at least a few Gyr. Based on these lines of argument, $\mathrm{BD}+20307$ is not a very young system, and the huge amount of warm dust orbiting the close binary has no direct relationship with the era of planet formation. The collision that created the dust must have been recent. Indeed, the massive amount of dust points toward the presence of terrestial planets, which argues that BD +20 307 is the first known example of planets orbiting a close binary star.

\section{ACKNOWLEDGMENTS}

This research was supported in part by NASA through a Chandra Observatory award to UCLA. Automated astronomy at Tennessee State University is supported by NASA, NSF, Tennessee State University, and the State of Tennessee through its Centers of Excellence program.

\section{REFERENCES}

1. J. H. Rhee, I. Song, and B. Zuckerman, Astrophys. J. 675, 777-783 (2008).

2. I. Song, B. Zuckerman, A. J. Weinberger, and E. E. Becklin Nature 436, 363-365 (2005)

3. A. J. Weinberger Astrophys. J. 679, L41-L44 (2008). 